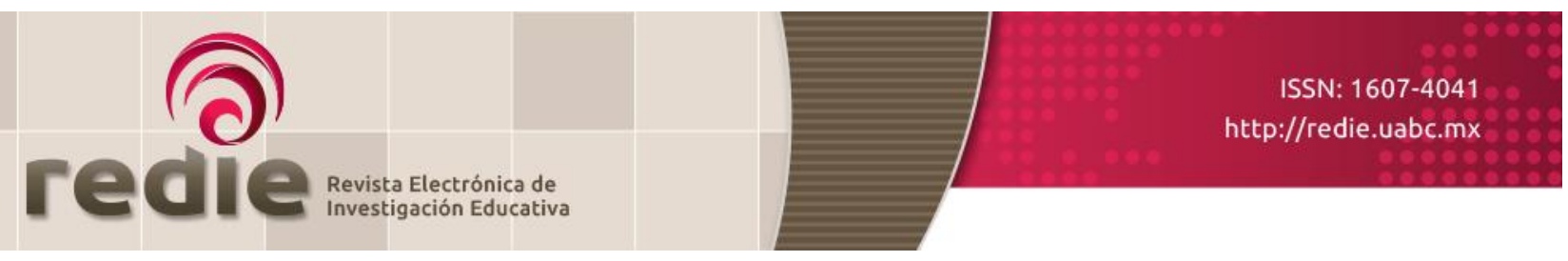

Vol. 21, 2019/e06

\title{
El valor de la escolarización en estudiantes de preparatorias de élite de la Ciudad de México
}

\section{The Value of Schooling for Elite High School Students in Mexico City}

\author{
Juan Espíndola Mata (*) juan.espindola@cide.edu \\ Blanca Heredia Rubio (*) heredia.blanca@gmail.com \\ Marisol Vazquez Cuevas (*) marisol.vazquez@cide.edu \\ (*) Centro de Investigación y Docencia Económicas \\ (Recibido: 21 de febrero de 2017; Aceptado para su publicación: 18 de agosto de 2017)
}

Cómo citar: Espíndola, J., Heredia, B. y Vazquez, M. (2019). El valor de la escolarización en estudiantes de preparatorias de élite de la Ciudad de México. Revista Electrónica de Investigación Educativa, 21, e06, 1-11. doi:10.24320/redie.2019.21.e06.1833

\section{Resumen}

Este trabajo examina el valor de la escolarización en estudiantes de preparatorias de élite de la Ciudad de México. En primer lugar, el artículo documenta el hecho de que el rendimiento académico de estos alumnos es bajo si se le compara con el de estudiantes de nivel socioeconómico similar en otros países, y si se toman como base de evaluación sus resultados en pruebas estandarizadas nacionales. Obviamente, el rendimiento académico poco satisfactorio de estos estudiantes, que asisten a instituciones privadas con recursos abundantes, no puede atribuirse a la precariedad económica y la consiguiente falta de oportunidades educativas. En el documento se explora la hipótesis de que para los jóvenes de este círculo social, que cuentan con un vasto patrimonio heredado, el mérito y el talento educativo no juegan un papel central en su concepción de desarrollo profesional o de vida. A efecto de sustentar esta hipótesis, se examina el valor que tanto padres de familia como directores de escuelas particulares de élite atribuyen a la escolaridad.

Palabras clave: Enseñanza privada, escuelas privadas, escolarización, clase social.

\section{Abstract}

This paper examines the value of schooling for students of elite high schools in Mexico City. The study begins by documenting the fact that these students' academic performance is poor in comparison to students of similar socio-economic status in other countries, and on the basis of their results in standardized national tests. Clearly, such unsatisfactory academic performance by these students, who attend private institutions with a wealth of resources, cannot be attributed to financial hardship and a resulting lack of educational opportunities. This work explores the hypothesis that, for young students from this social circle - who enjoy vast inherited wealth - educational merit and talent do not play a key role in their conception of life or professional development. To support this hypothesis, this study explores the value placed on schooling both by parents and the directors of elite private schools. 


\section{Introducción}

Como lo muestran diversas pruebas estandarizadas de rendimiento académico, como el Programa Internacional de Evaluación de Estudiantes (PISA, por sus siglas en inglés), el desempeño educativo de los estudiantes mexicanos es notablemente más bajo que el de los estudiantes de otros países. Más allá del bajo rendimiento comparativo del estudiante mexicano promedio, sin embargo, llama la atención un dato en particular: si se consultan los datos por nivel socioeconómico y se examina a los estudiantes provenientes de los sectores de mayores ingresos, la comparación es aún más desfavorable para los alumnos mexicanos. Así, por ejemplo, en el área de Matemáticas de PISA 2012, el puntaje promedio de los estudiantes del decil de ingreso más alto de todos los países la OCDE fue de 554, mientras que en el caso de México fue de 464. En otras palabras, los alumnos mexicanos más ricos se ubican alrededor de dos grados escolares por debajo de sus homólogos dentro de la OCDE. Si bien los alumnos mexicanos de menores ingresos tampoco tuvieron un buen rendimiento en comparación con sus pares de otros países, la distancia entre sus resultados en Matemáticas (376 puntos) y el promedio obtenido por los alumnos de menores ingresos en el conjunto de los países de esa organización (436) fue, con todo, menor a la de sus compatriotas más pudientes económicamente vis a vis los alumnos del decil de ingreso más alto de la OCDE. Específicamente, poco más de un año de escolaridad en el caso de los alumnos en desventaja económica frente a los dos grados escolares que separan a nuestros estudiantes más ricos de sus pares en la OCDE (OCDE 2011).

Este trabajo examina el valor de la escolarización en estudiantes de preparatorias de élite de la ciudad de México. En primer lugar, el artículo documenta el hecho de que el rendimiento académico de estos alumnos es bajo si se le compara con el de estudiantes de nivel socioeconómico similar en otros países, y si se toman sus resultados en pruebas estandarizadas como base de evaluación. Obviamente, el rendimiento académico poco satisfactorio de estos estudiantes, que asisten a instituciones privadas con recursos abundantes, no puede atribuirse a la precariedad económica y su consiguiente falta de oportunidades educativas. En este documento exploramos la hipótesis de que para los jóvenes de este círculo social, que cuentan con un vasto patrimonio heredado, el mérito y el talento educativo no juegan un papel central en su concepción sobre el desarrollo profesional o de vida. A efecto de sustentar esta hipótesis, examinamos el valor que tanto padres de familia como directores de escuelas particulares de élite atribuyen a la escolaridad. Muchas de las escuelas particulares en las que estudian los hijos de las élites políticas y económicas del país no tienen como su tarea principal fomentar el talento de sus alumnos. Su misión es dotarlos de capital social, ofrecerles un espacio de distinción social e inculcarles ciertos valores morales y religiosos.

Llevar a cabo este tipo de análisis es de suma importancia por al menos una razón: el estado de la investigación sobre la educación básica y media superior de los sectores de altos ingresos en México es prácticamente inexistente, incluso la investigación sobre el caso latinoamericano es escasa (Gubbins, 2014; Tiramonti y Ziegler 2008; Ziegler y Gessaghi 2012). En México, con la excepción de algunas obras de corte histórico, el tema no ha sido explorado de manera sistemática y rigurosa, y la mayoría del trabajo académico sobre educación privada disponible se concentra en el nivel superior. El presente artículo busca contribuir a llenar esta laguna.

Vale la pena hacer una precisión sobre el sector educativo privado en la Ciudad de México antes de iniciar. Según datos de la Secretaría de Educación Pública (SEP), durante el ciclo escolar 2012-2013, en la capital del país, 23\% de los estudiantes de todos los niveles (de educación básica a media superior) estudian en escuelas privadas, y $77 \%$ lo hace en escuelas públicas. En el nivel medio superior en específico, $20 \%$ de los alumnos estudia en escuelas privadas y el resto en escuelas públicas (SEP, 2014). Ahora bien, no son pocas las diferencias al interior del sector privado. Las escuelas particulares en México distan mucho de ser un sector homogéneo. Para empezar, el origen de todas ellas no es el mismo. Las escuelas particulares en México, y particularmente en la capital, nacen por muy variadas razones. Algunas surgen para compensar la incapacidad del Estado para atender la demanda educativa; otras lo hacen como respuesta a la baja calidad del sistema educativo público. Muchas familias buscan en esas escuelas lo que, desde su perspectiva, es un estándar de calidad educativa mayor al ofrecido por las escuelas oficiales. En otros casos, las escuelas particulares surgen para impartir algún tipo de instrucción que las oficiales no ofrecen, como la enseñanza de valores religiosos. De hecho, la irrupción más significativa de colegios privados en 
el país ocurrió como reacción al espíritu laicista -luego, durante el Cardenismo, claramente socialista, del proyecto educativo posrevolucionario (Lerner, 1979; Torres, 2004). Por último, otros colegios abren sus puertas para preservar ciertas identidades étnicas o culturales. Así, las escuelas privadas en México constituyen un universo heterogéneo.

\section{Antecedentes}

Estudios recientes han puesto en entredicho la idea que las escuelas privadas son las mejores formadoras de estudiantes (Belfield y Levin, 2005; Levin 2001; Levin y Belfield 2003; Lubienski y Theule, 2014; para el caso mexicano, Chávez 2010); también han puesto en duda la creencia de que la llamada "ansiedad de clase media" promueva el alza en los estándares académicos de las escuelas privadas (Labaree, 1997).

Uno de los hallazgos más importantes del trabajo de Benveniste, Carnoy y Rothstein (2003) sobre escuelas privadas y públicas en Estados Unidos es que las primeras no son necesariamente mejores que las segundas. A contrapelo de la idea de que al competir entre sí por estudiantes, las escuelas, como cualquier otro producto del mercado, mejorarán su calidad; en el caso de la educación -según estos autores- muchos de los supuestos del modelo de libre mercado no se cumplen. Además de que las escuelas públicas son más sensibles a las exigencias y necesidades de los padres de lo que ese modelo supone; por su parte, las escuelas privadas (foco de nuestra atención) son menos receptivas a las exigencias de los padres de lo que el mismo modelo anticiparía.

Las autoridades de muchas de estas escuelas consideran que su misión es definir un método de enseñanza y cierto currículo al margen de las preferencias parentales, pues desde su punto de vista son los directivos y maestros de la propia escuela, no los padres de familia, quienes tienen el expertise para definirlos. Y no les falta razón: en muchos casos, cuando los padres de familia irrumpen en el proceso escolar, el resultado no siempre es la mejora educativa. La intervención parental puede conducir a que se vulnere y manipule el proceso pedagógico y, además, en muchas ocasiones lo que buscan los padres de familia son instituciones educativas que otorguen credenciales y títulos "mercadeables" que no siempre guardan correspondencia con altos estándares académicos. Más aún, a diferencia de lo que estipula el modelo de libre mercado, en los hechos son las escuelas privadas las que "escogen" a sus alumnos y no a la inversa. Finalmente, al evaluar escuelas, los padres de familia valoran otras dimensiones, además de la académica; en las escuelas católicas y militares, por ejemplo, privilegian la instrucción religiosa y la disciplina, respectivamente, sobre la formación académica. Así, ni la escuela privada es inherentemente mejor que la pública ni la ansiedad parental descrita antes se traduce necesariamente en mejores resultados académicos.

Рara el caso mexicano parece ser más importante, como marco interpretativo de la situación de las escuelas privadas, el paradigma elaborado por los teóricos de la reproducción social. Para autores como Bourdieu y Passeron (1996), la educación juega un papel como reproductora de la cultura, la estructura social y la económica a través de "estrategias de clase". Dichas estrategias se refieren a que determinados grupos sociales (las élites culturales, por ejemplo) impulsan ciertos contenidos académicos (humanidades) y no otros (estudios con orientación profesional). Pero las estrategias de clase también pueden referirse de manera aún más elemental a la valoración que se da, y a la actitud que se muestra frente al estudio en general. Como es sabido, Bourdieu y Passeron, en particular el primero, fueron los primeros teóricos sociales en desarrollar los conceptos de "capital cultural" y "capital social". Estos conceptos, ampliamente difundidos en la literatura académica contemporánea se refieren, el primero, al conocimiento y las habilidades necesarias para conseguir el acceso al mercado de trabajo, y el segundo, a las "conexiones" y redes sociales. A partir de estos conceptos, y basados en estudios longitudinales de estudiantes de Harvard y Yale, Zweigenhaft (1993) argumenta que los estudiantes de bachilleratos públicos superan académicamente a los de escuelas privadas de élite. Su explicación, que es lo que interesa resaltar para propósitos de este trabajo, es que esto se debe a que los primeros buscan acumular capital cultural, mientras que los segundos, por el contrario, invierten en capital social. 
Para aquellos que ya están en la cima de la estructura de clase, las relaciones con otros sujetos que también tendrán poder es seguramente más importante para ellos que tener un desempeño académico excepcional. (Zweigenhaft, 1993, p. 212).

Algo similar afirma Ambrose (2009) al referirse a la "opulencia superficial pasiva" (passive shallow opulence). Según la autora, este fenómeno se refiere a la actitud de "individuos privilegiados que aceptan con complacencia las cómodas ventajas de la riqueza y el estatus" y, en consecuencia, "desarrollan aspiraciones y talentos limitados" (Ambrose 2009, 888). Creemos que algo muy similar ocurre en México, y este trabajo es un primer esfuerzo para documentar esa hipótesis.

\section{Un acercamiento cuantitativo}

En este apartado se identifican algunas de las escuelas particularmente costosas que no destacan por obtener resultados académicos sobresalientes. Se examinan estos casos porque van en contra de una de las regularidades más asentadas en los estudios sobre educación: a mayor ingreso, mejores rendimientos académicos del estudiante.

\subsection{Análisis de la calidad educativa basada en el puntaje ENLACE 2009}

Se analiza aquí la relación que existe entre el costo y la calidad de las escuelas preparatorias privadas de la Ciudad de México, para esto, el costo de las escuelas se mide a través de la colegiatura mensual, mientras que la calidad se mide a través de los resultados de la prueba ENLACE 2009. Sólo se consiguieron los costos de la colegiatura de 67 escuelas a través de diversas fuentes, entre ellas una encuesta del periódico Reforma, que contempla una columna con el costo de la colegiatura por escuela, así como llamadas telefónicas para verificar dichos datos. Se procedió de esta manera porque en varias escuelas se mostraron reacios a compartir información, sólo la ofrecen a padres de familia interesados. Esta es la razón por la cual la muestra es limitada, aunque los montos incluidos son confiables.

¿Existe una relación entre el costo y la calidad de las escuelas privadas de la Ciudad de México? Para responder a esta pregunta se requiere información de la colegiatura de las escuelas y de los puntajes obtenidos en ENLACE que permitan medir la calidad. La muestra empleada en el análisis se construyó en dos etapas: primero se utilizó la base de datos que incluye todas las preparatorias privadas en el Distrito Federal; de 446 escuelas disponibles sólo 67 contaban con información sobre la colegiatura. Posteriormente, entre dichas escuelas se buscó que hubiesen sido evaluadas con la prueba ENLACE. De las 67 escuelas, sólo 30 participaron en la prueba ENLACE 2009, pues aunque es obligatoria para los centros escolares públicos, hasta hace poco era optativa para las escuelas privadas. Cabe mencionar que se seleccionó este año de la prueba debido a que de 2008 a 2013, el 2009 fue el año en el que participaron más escuelas privadas. En esta sección se analiza la calidad educativa de una selección de escuelas privadas de la Ciudad de México con base en los puntajes promedio obtenidos en la prueba ENLACE 2009. La tabla I presenta el ranking por promedio de habilidad lectora y habilidad matemática.

\footnotetext{
${ }^{1}$ La Evaluación Nacional de Logro Académico en Centros Escolares (ENLACE) es una prueba del Sistema Educativo Nacional que se aplica a planteles públicos y privados en México.
} 
Tabla I. Ranking por promedio en habilidad Lectora y habilidad Matemática

\begin{tabular}{|c|c|c|c|c|}
\hline Preparatoria & $\begin{array}{c}\text { Puntaje } \\
\text { Promedio } \\
\text { (Lectura) }\end{array}$ & Nivel & $\begin{array}{c}\text { Puntaje } \\
\text { Promedio } \\
\text { (Mat.) }\end{array}$ & Nivel \\
\hline Colegio Cristóbal Colón & 1573.35 & Excelente & 1948.56 & Bueno \\
\hline La Escuela de Lancaster & 1554.15 & Excelente & 1595.62 & Bueno \\
\hline Preparatoria UnITEC Sur & 1509.68 & Excelente & 1031.80 & Elemental \\
\hline Centro de Educación y Cultura Ajusco & 1465.57 & Excelente & 1219.48 & Bueno \\
\hline Escuela Tomás Alva Edison & 1403.74 & Bueno & 1389.09 & Bueno \\
\hline Instituto Irlandés & 1396.25 & Bueno & 1751.45 & Bueno \\
\hline Colegio Ciudad de México & 1366.85 & Bueno & 33473.37 & Excelente \\
\hline U. Panamericana Preparatoria Yaocalli Femenino & 1334.70 & Bueno & 1588.57 & Bueno \\
\hline Instituto Highlands & 1297.50 & Bueno & 2177.74 & Bueno \\
\hline Colegio Bilbao & 1266.64 & Bueno & 1186.16 & Bueno \\
\hline Instituto Educativo Olinca & 1226.48 & Bueno & 1710.20 & Bueno \\
\hline Colegio Oxford Bachillerato Femenino & 1225.10 & Bueno & 946.27 & Elemental \\
\hline Colegio Británico & 1166.63 & Bueno & 475.47 & Elemental \\
\hline Instituto Cumbres de Vista Hermosa & 1066.47 & Bueno & 1327.74 & Bueno \\
\hline Preparatoria unItEC Atizapán & 1057.22 & Bueno & 1167.24 & Elemental \\
\hline Colegio Merici & 1010.79 & Bueno & 1272.70 & Bueno \\
\hline The American School Foundation & 1004.28 & Bueno & 1520.78 & Bueno \\
\hline U. Panamericana Preparatoria Masculino & 958.78 & Bueno & 1143.78 & Elemental \\
\hline Instituto Oxford Masculino & 936.21 & Bueno & 569 & Elemental \\
\hline ITESM Preparatoria & 874.19 & Bueno & 1290.23 & Bueno \\
\hline Colegio Alemán Alexander Von Humboldt & 857.34 & Bueno & 1140.58 & Elemental \\
\hline Colegio México Bachillerato & 817.61 & Bueno & 770.16 & Elemental \\
\hline Academia Maddox & 614.87 & Bueno & 660.95 & Elemental \\
\hline Universidad Intercontinental Bachillerato & 593.66 & Bueno & 497.37 & Elemental \\
\hline Preparatoria UNITEC Marina & 534.52 & Bueno & 571.49 & Elemental \\
\hline Colegio del Bosque & 389.06 & Bueno & 19819.55 & Excelente \\
\hline Preparatoria unITEc Ecatepec & 357.90 & Bueno & 883.95 & Elemental \\
\hline Universidad Latina Preparatoria & 333.16 & Bueno & 8909.93 & Excelente \\
\hline Colegio Williams & 129.28 & Bueno & 51.24 & Elemental \\
\hline La Salle Simón Bolívar & -226.669 & Elemental & -43.38 & Elemental \\
\hline
\end{tabular}

Elaboración propia con información de ENLACE (2009).

Como se puede observar en la tabla I, en habilidad lectora las escuelas privadas participantes en ENLACE 2009 obtuvieron puntajes promedio que las ubican, a la mayoría, en los niveles bueno y excelente. Sin embargo, en habilidad matemática los resultados son totalmente diferentes, ya que $46 \%$ de las escuelas de la muestra obtuvieron resultados elementales en la prueba. Este hallazgo no era el esperado, ya que se pensaría que las escuelas con colegiaturas tan altas tendrían puntajes mucho más elevados en todas las áreas, lo que pone en duda la calidad de la educación que imparten instituciones de renombre y que cobran altas colegiaturas. El hallazgo sugiere también, por otra parte, que la influencia del estatus socioeconómico parece ser mayor para el logro en lectura que para el nivel de logro en matemáticas. Las escuelas que muestran resultados atípicos son: Colegio Ciudad de México, Colegio del Bosque y Universidad Latina Preparatoria, las tres con un puntaje muy superior en matemáticas comparado con el de lectura. Por otra parte, las demás preparatoria cuentan con puntajes "congruentes" entre ambas habilidades.

De acuerdo con la tabla I, se puede observar que el Colegio Cristóbal Colón es el que tiene mayor puntaje en habilidad lectora y, además, está entre los mejores en habilidad matemática. Aunado a esto, el costo de la mensualidad es menor a los 10,000 pesos y la inscripción asciende a 11,490 pesos. Por otra parte, La Salle Simón Bolívar y el Colegio Williams son las escuelas con peores puntajes en ambas habilidades medidas por ENLACE 2009; sin embargo, La Salle Simón Bolívar es significativamente más barata que el Colegio Williams. La preparatoria más cara, The American School Foundation, obtiene puntajes bajos en 
ambas pruebas -17 en habilidad lectora y 10 en habilidad matemática.

\subsection{Análisis de la relación entre calidad y costo}

En este análisis se compara la calidad educativa -medida a través de la prueba ENLACE 2009- de las 30 escuelas antes presentadas con su costo educativo -medido a través de la colegiatura mensual.

A continuación se presentan las 10 escuelas, de la selección aquí considerada, que obtuvieron el puntaje más alto en la prueba ENLACE 2009 -medida a través de la habilidad lectora y matemática- así como las que tienen los costos más altos de colegiatura.

Tabla II. Escuelas con mejor puntaje en ENLACE 2009 y con mayor costo de colegiatura

\begin{tabular}{|c|c|c|c|}
\hline & \multicolumn{2}{|c|}{ Preparatorias con mejor puntaje } & \multirow{2}{*}{ Preparatorias más costosas } \\
\hline & Lectura & Matemáticas & \\
\hline 1 & Colegio Cristóbal Colón & Colegio Ciudad de México & The American School Foundation \\
\hline 2 & La Escuela de Lancaster & Colegio del Bosque & Colegio Británico \\
\hline 3 & Preparatoria unITEC Sur & Universidad Latina Preparatoria & $\begin{array}{l}\text { Colegio Alemán Alexander Von } \\
\text { Humboldt }\end{array}$ \\
\hline 4 & $\begin{array}{l}\text { Centro de Educación y } \\
\text { Cultura Ajusco }\end{array}$ & Instituto Highlands & Instituto Highlands \\
\hline 5 & Escuela Tomás Alva Edison & Colegio Cristóbal Colón & Instituto Educativo Olinca \\
\hline 6 & Instituto Irlandés & Instituto Irlandés & Colegio Ciudad de México \\
\hline 7 & Colegio Ciudad de México & Instituto Educativo Olinca & Preparatoria unITEc Ecatepec \\
\hline 8 & $\begin{array}{l}\text { U. Panamericana Preparatoria } \\
\text { Yaocalli Femenino }\end{array}$ & La Escuela de Lancaster & Instituto Cumbres de Vista Hermosa \\
\hline 9 & Instituto Highlands & $\begin{array}{l}\text { U. Panamericana Preparatoria } \\
\text { Yaocalli Femenino }\end{array}$ & Universidad Intercontinental Bachillerato \\
\hline 10 & Colegio Bilbao & The American School Foundation & $\begin{array}{l}\text { U. Panamericana Preparatoria Yaocalli } \\
\text { Femenino }\end{array}$ \\
\hline
\end{tabular}

Elaboración propia con información de ENLACE (2009).

En la tabla II se observa que el listado de las 10 escuelas con mejores puntajes en ENLACE 2009 en lectura y matemáticas no coincide con el listado de las 10 escuelas más caras de la Ciudad de México. A pesar de que el Colegio Cristóbal Colón obtuvo el mejor puntaje en lectura, no entra en el ranking de las escuelas más caras. El Colegio Ciudad de México obtuvo el mejor puntaje en matemáticas y ocupa el sexto lugar en el ranking de las escuelas más caras. Por último, The American School Foundation es la escuela más cara, a pesar de ello ocupa la décima posición entre las 10 escuelas más caras de la capital del país en lo que hace a su puntaje en ENLACE matemáticas, mientras que en lectura no aparece dentro de las 10 mejores.

\section{Un acercamiento cualitativo}

Si como el apartado tres, el desempeño académico no parece ser una prioridad para las escuelas particulares de élite, entonces: ¿qué otras consideraciones orientan su misión?, ¿cuál es la valoración que dan a la escolarización, a la promoción del talento y a la preparación académica en general?

Para la elaboración de este trabajo se utilizaron dos herramientas antropológicas: observación participante y conversaciones grupales e individuales a profundidad. Los reportes de observación participante consisten en observar y registrar a detalle los fenómenos sociales en sus contextos, interacciones y diálogos, detectando los factores y variables más relevantes para cubrir y explicar los objetivos de la investigación. Las conversaciones grupales a profundidad consisten en pláticas que los investigadores sostienen con un grupo de personas a través de ejes temáticos de interés, y que fluyen gracias al rapport que éstos han podido establecer previamente con las personas. El objetivo de esta técnica es profundizar en los temas de interés a partir de las contradicciones, regularidades o ausencias de información que vayan surgiendo en los discursos de los participantes, lo que permite identificar - 
desde una perspectiva sociocultural- las aristas del fenómeno estudiado.

En consonancia con estas herramientas metodológicas, por un lado se llevó a cabo una serie de encuentros con siete directivos de algunas escuelas particulares, y con 36 madres y padres de familia, por otro. ${ }^{2}$ Los encuentros tuvieron lugar entre octubre y diciembre de 2014 en el Distrito Federal. Luego se convocó a una reunión con padres de familia de clases privilegiadas, los participantes fueron elegidos tanto en función de la escuela a la que asisten sus hijos o en la que laboran (se trata en todos los casos de escuelas de élite) como en función de su disponibilidad. ${ }^{3}$ Tanto en el caso de las entrevistas como en el de la reunión, el propósito fue entender la valoración que los directivos de las escuelas y los jefes de familia dan al talento académico y, en general, comprender qué buscan (y qué ganan) estos alumnos al ingresar a estas instituciones.

Una aclaración, antes de documentar los principales hallazgos de las entrevistas y las observaciones. Al hablar de clases "acomodadas" o privilegiadas nos referimos, de manera muy general, a todo el espectro de las clases medias: típica, media alta y alta. Tal diferenciación se establece no sólo a partir de un rango de ingresos determinado, dentro del cual todas las familias serían consideradas como de clase media, sino también en función de un conjunto de códigos o set de valores que pueden variar dentro de la clase media: desde las tradiciones que reafirman sus valores, la educación como mecanismo de movilidad social (sea formal o informal), hasta una cultura generalizada del valor del esfuerzo. Así, estamos conscientes de que cabe hacer diferenciaciones al interior de las clases medias. Para los fines de este trabajo, sin embargo, al hablar de ellas no se establecen diferencias, aunque se harán precisiones cuando sea necesario.

A partir de las entrevistas y observaciones mencionadas, pudo determinarse, a grandes rasgos, el valor social que la institución escolar tiene para los miembros de las clases acomodadas. Entre las madres de familia, el valor de la escuela estriba en su capacidad para construir un espacio donde los alumnos puedan reproducir ciertas prácticas socioculturales y estilos de vida. En palabras de una de las madres: "Te da cierta seguridad que [en la escuela tus hijos] se lleven con gente de papás que piensan más o menos como tú, sea a nivel religioso, sea a nivel laboral, en lo personal, sea a nivel de valores sociales". Las escuelas son fundamentales en tanto que les permiten tanto a hijos como a padres reafirmar su diferencia social con respecto a otras clases sociales; de hecho, desde la perspectiva valorativa de las madres de familia, particularmente las de clase media típica, la escuela que es "demasiado" incluyente se devalúa.

La aportación más importante de las entrevistas y el encuentro fue la determinación y jerarquización de los "activos" o "bienes" que los padres de familia persiguen al inscribir a sus hijos en escuelas privadas. Estos actores (familiares, directivos) identificaron explícitamente tales bienes y su jerarquía en los siguientes términos:

1. Relaciones sociales. En las entrevistas, muchas madres reconocieron que, puestas a elegir entre una escuela de calidad académica pero sin espacios para que sus hijos convivan con pares de su mismo nivel socioeconómico, y una escuela de menor calidad pero donde se promueven dichos espacios, la segunda opción es más atractiva.

Hablando sobre la educación de sus hijos, una madre de familia puntualizó:

Yo no sé cuánto la cultura en México es de meritocracia... yo creo que muchos chavos y chavas de familias privilegiadas no se preocupan porque van a encontrar la palanca para entrar a donde quieran o van a heredar y yo creo que la meritocracia pesa muy poco.

Aquí el punto sobre la importancia de las redes sociales se hace a manera de crítica. No obstante, el posicionamiento de la madre frente al problema -la inexistencia de una cultura meritocrática en México-

\footnotetext{
${ }^{2}$ Queda pendiente entrevistar también a los alumnos, dichas entrevistas serían parte de un futuro artículo.

${ }^{3}$ Todas las personas contactadas para realizar este trabajo autorizaron el uso de la información que aportaron. Por consideraciones de corte ético, y a petición de ellos, se mantiene el anonimato.
} 
no es el repudio activo, por decirlo de alguna manera, sino la resignación. ${ }^{4}$

Más allá del discurso de las madres y los directores, vale la pena incluir otro indicador sobre la importancia que algunas escuelas particulares adscriben, para beneplácito de los padres de familia, al fomento de las relaciones sociales. Nos referimos a las estrategias de promoción de algunas de ellas. Los videos promocionales del Bachillerato Anáhuac, es decir las preparatorias vinculadas a la Legión de Cristo, son un buen ejemplo. En uno de esos videos, en donde se entrevista a diversos miembros de la comunidad de esas escuelas (alumnos, padres de familia y autoridades) es unánime la opinión sobre una de las (¿o la?) fortaleza de la "educación legionaria": el valor de la amistad (léase relaciones sociales). En el video, un estudiante dice abiertamente que una de las grandes ventajas de su escuela fue que le permitió "crecer en un ambiente de gente afín a ella." En la misma tesitura, un padre de familia afirma que la escuela es el lugar donde se "reafirman las amistades." En su valoración hay un tono más prescriptivo que descriptivo. En su voz, el afianzamiento de las amistades se plantea casi como uno de los imperativos de la misión escolar. De la experiencia en la escuela, dice este padre, "es muy importante vivirla con amigos, que viven lo mismo que tú", y añade que en su caso, "los que están junto a mí todos los días son mis amigos de prepa". De ahí la importancia de que sus hijos, concluye en el mismo video una madre de familia, "de acabar la escuela donde la empezaron" (Bachillerato Anáhuac, 2012). La segregación social hecha virtud y presentada como estrategia de marketing.

2. Promover un sistema de valores religiosos y/o morales. Esto lo pone en los siguientes términos una madre cuya hija está inscrita en el Instituto Asunción, escuela de la congregación de las religiosas de la Asunción (a la cual, por cierto, asistieron los hijos del expresidente Felipe Calderón):

Yo creo que debe guardar un equilibrio, sólo enfocarte a lo académico sería un error, sólo enfocarte a lo formativo sería un error, yo creo que la escuela es un proyecto de educación integral y se forman más, a mí lo que me gusta es que se forman mucho los niños en la especialidad social, les generan desde chicos una responsabilidad y un compromiso social, que eso urge en México, les dan su tiempo para gente que lo necesita... tienen valores, tienen religión, creo que un equilibrio es como todo en la vida... El alumno es buen alumno donde lo pongas, entonces también puede ser la mejor escuela académicamente y tu hijo un desastre o puede ser la peor escuela, pero tu chavo es súper inteligente y la va a aprovechar.

Los directores de varias escuelas particulares argumentaron en un sentido muy similar. El director de una estas escuelas, de orientación religiosa, también hizo hincapié en promover en su escuela una "educación de tipo humanista" con "conciencia y sensibilidad social." A su vez, el director de una preparatoria de perfil similar habla de una educación integral en los siguientes términos:

Son chavos [los estudiantes de la preparatoria] muy sensibles a los temas sociales, son muy buenos en Matemáticas, son chavos con una visión ética muy alta. Son buenos hombres con una exigencia académica que es el verdadero talento, el verdadero talento de la prepa. Son chavos bien preparados y con una formación humana que se nota en otras universidades.

Cuestión aparte es si los colegios de los que se ha hablado, y otros similares, consiguen inculcar tales valores en sus alumnos. Por ejemplo, durante las conversaciones con las madres de familia, algunas de las percepciones sociales ampliamente extendidas sobre los colegios de los Legionarios afloraron en la discusión. Según estas percepciones, dichos colegios descuidan tanto el aspecto académico como la formación en valores en aras del lucro. Una madre lo puso en los siguientes términos:

Sí siento que tienen muchas debilidades los Legionarios, yo lo metí ahí sobre todo porque se supone que tenía un maravilloso inglés y sí salió con un buen inglés, pero sí tienen muchas deficiencias, no hace nada por arreglar, las reglas no se cumplen, influye mucho el valor del dinero, tienen muchos problemas en cuanto a la relación; si tienes lana te llevas con los que tienen lana... los profesores son todólogos, igual te daban Matemáticas que Historia que

\footnotetext{
${ }^{4}$ El periodista Ricardo Raphael (2014) llega a una conclusión similar a la nuestra; según él, en las escuelas mexicanas importa más la adquisición de "conocidos" que la de "conocimientos."
} 
Geografía... no tiene el nivel que yo hubiera querido, yo veía el nivel que tenía el cum, incluso el Sagrado, el Asunción, para mí no hay escuela ideal, todas tienen sus pros y sus contras... a mi hija sí la tuve que sacar.

3. Robustecer identidades comunitarias. Es el caso de las escuelas judías. Algunas madres afirmaron sin ambages: "Mi esposo y yo dudamos si inscribirlo al Colegio Hebreo Sefaradí o al Americano. Al final prevaleció nuestro deseo de reforzar su vinculación con la comunidad y la tradición judía". Esta dimensión de las expectativas de los padres de familia no es ninguna sorpresa, forma parte del repertorio cultural de las comunidades judías en todo el mundo y responde a causas históricas de sobra conocidas.

Obviamente, todo lo anterior no quiere decir que los padres de familia o los directores de las escuelas particulares descuiden por entero el ámbito académico y la promoción del talento. Muchos de ellos, por ejemplo, ven como criterio de excelencia académica el hecho de que las escuelas de sus hijos, en el caso de las madres, o las que ellos dirigen, en el caso de los directores, ofrezcan la posibilidad de cursar el Bachillerato Internacional o, en el caso del Colegio Alemán, de obtener el certificado terminal de la preparatoria alemana, el Abitur. En otras palabras, estos colegios buscan ampliar el capital cultural de sus estudiantes a través del desarrollo de habilidades para estudios en el extranjero. Otras preparatorias, como la de la Universidad Panamericana, o el Colegio Americano, promueven el talento musical a través del apoyo a una orquesta escolar, en el primer caso, y el talento artístico a través del apoyo a actividades como las artes plásticas o la escritura, en el segundo. Escuelas menos orientadas hacia sistemas internacionales de reconocimiento académico, como la propia Universidad Panamericana, tienen el mérito de haber recibido el cuarto puntaje más alto en la parte matemática de la prueba ENLACE en el Distrito Federal, sólo detrás de dos vocacionales del Politécnico y la Fundación Azteca.

Algunas preparatorias tienen criterios de ingreso más o menos rigurosos, y sus directores consideran que este "filtro" es el primer componente de su política de talento. Así, una de estas preparatorias exige un puntaje de 1,085 en el examen del Centro Nacional de Evaluación para la Educación Superior (Ceneval) y un promedio de 7.5 (además de un examen psicométrico y otro de las circunstancias familiares de cada alumno) para aceptar a sus alumnos. Esto contrasta con las políticas de ingreso de otras escuelas, en las cuales el criterio de ingreso más importante es el poder adquisitivo de las familias.

Por otra parte, llama la atención que muchas de estas escuelas decidan evaluar y promover el talento de sus estudiantes por medio de actividades (talleres, competencias, pruebas) que ellas mismas instituyen, al margen de los eventos nacionales encaminados a ese fin. Por ejemplo, muchas escuelas particulares optan por no participar en las Olimpiadas nacionales de Matemáticas, o tomar con seriedad la prueba ENLACE (la cual, reiteramos y sin que quede muy claro por qué, es obligatoria para las escuelas públicas рero opcional para las privadas), y en cambio fomentan entre sus estudiantes eventos y mecanismos de evaluación académica organizados por las propias escuelas, o de corte internacional. Por ejemplo, la red de Colegios de la Legión de Cristo, organiza desde 1996 el Premio Internacional Bachillerato Anáhuac, dirigido a todos los alumnos del Bachillerato de su red de colegios en diversas áreas, como Matemáticas, Creación literaria, Debate y Robótica, entre otras. La razón (declarada) para no participar en competencias de habilidades nacionales es que éstas no suelen ser genuinamente competitivas y están sesgadas a favor de alumnos de escuelas públicas.

Es interesante apuntar que algunas de las prácticas de apoyo al talento que promueve un grupo de las preparatorias analizadas en este trabajo son al mismo tiempo proyectos educativos de apoyo a grupos de bajos ingresos. La preparatoria de la Universidad Panamericana, por ejemplo, mantiene un turno vespertino (el grupo F) para estudiantes de bajos recursos. Por su parte, los Legionarios sostienen una red de escuelas llamadas Mano Amiga, las cuales cuentan con 22 colegios y 14,000 alumnos en toda la república. El ingreso a estos centros de estudio, lo mismo que al grupo $\mathrm{F}$ de la Panamericana, se da en función de una combinación de criterios meritocráticos y socioeconómicos. Estos esquemas de apoyo, sin embargo, replican el espíritu de segregación del sistema público/privado. 
Cabe mencionar que los hallazgos de este artículo matizan las versiones más radicales sobre la educación de las clases privilegiadas en México. Según esa creencia, los hijos de las élites mexicanas han crecido en un ambiente adverso a la superación por la vía intelectual; esos jóvenes, según esta perspectiva, compartirían la percepción de que el estudio es inútil. Su contexto cultural estaría regido por normas sociales de acuerdo con las cuales es fuente de prestigio social ostentarse como descendiente de una familia que puede vivir con holgura sin estudiar ni trabajar; dicho contexto incluiría también convenciones de género según las cuales las mujeres no pueden destacar intelectualmente si desean venderse en el mercado de matrimonios. Todo ello es válido para un sector reducido dentro del ya por sí reducido ámbito de las élites socioeconómicas; para el resto de dichas élites, el conocimiento académico -más que despreciarse- se relega en favor de otras consideraciones.

\section{Conclusión}

La investigación cuantitativa y cualitativa presentada hace suponer que la promoción del talento de los estudiantes, y en general la transmisión de conocimiento académico, no es el criterio prioritario ni para las escuelas particulares, al menos no para todas ellas, ni para los padres de familia que inscriben a sus hijos en ellas. Рагеciera ser que lo más importante es el acceso a una red de relaciones, algún tipo de certificación social o el fortalecimiento de identidades culturales. Estas valoraciones son perfectamente racionales desde el punto de vista de los actores en cuestión, una respuesta a las condicionantes del entorno.

Ante el predicamento de recibir instrucción de calidad o una extensa y densa red de relaciones (o cualquier otro de los criterios mencionados), la alternativa que tomen dichos actores dependerá de los incentivos que les ofrezca el contexto en el que están situados. Este resultado debería hacernos examinar críticamente el contexto social más general en el que están insertas las escuelas que han sido objeto de análisis. En una sociedad desigual, como la mexicana, donde una parte considerable de los márgenes de movilidad social no está determinada por el mérito, académico o profesional sino por el patrimonio heredado, la educación y la promoción del talento pierden importancia para los actores sociales. Esto no quiere decir que todos los miembros de las clases privilegiadas devalúen la actividad académica o que, en términos agregados, su rendimiento escolar sea más bajo que el de los alumnos de escuelas públicas. Que la dimensión académica pierda importancia simplemente quiere decir que en las decisiones educativas de los padres de familia intervienen criterios extra académicos.

\section{Referencias}

Ambrose, D. (2009). Large-scale socioeconomic, political, and cultural influences on giftedness and talent, en L. Shavinina (Ed.), International Handbook on Giftedness. Holanda: Springer.

Bachillerato Anáhuac. (2012). Spot promocional Bachillerato Anáhuac [video]. México. Disponible en http://vimeo.com/39430771

Belfield, C. y Levin, H. (2005). Privatizing educational choice: consequences for parents, schools, and public policy. Boulder, co: Paradigm Publishers.

Benveniste, L., Carnoy, M. y Rothstein, R. (2003). All else equal: are public and private schools different? Nueva York: Routledge Falmer.

Bourdieu, P. y Passeron, J. C. (1996). La Reproducción. Elementos para una teoría del sistema de enseñanza. México: Fontamara.

Chavez, F. W. (2010). Do children in private Schools learn more than in public schools? Evidence from Mexico. Munich Personal RePEc Archive, documento Núm. 30506. Recuperado de http://mpra.ub.unimuenchen.de/30506/ 
Evaluación Nacional del Logro Académico en Centros Escolares. (2009). Resultados de la prueba ENLACE 2009. Recuperado de http://enlace.sep.gob.mx/ba/resultados anteriores/

Gubbins, V. (2014). Estrategias educativas de familias de clase alta: Un estudio exploratorio. Revista Mexicana de Investigación Educativa, 19(63), 1069-1089. Recuperado de https://www.comie.org.mx/revista/v2018/rmie/index.php/nrmie/article/view/220/220

Labaree, D. (1997). How to succeed in school without really learning. New Haven, ст: Yale University Press.

Lerner, V. (1979). La educación socialista. México: El Colegio de México.

Levin, H. (2001). Privatizing education. Boulder, co: West view Press.

Levin, H. y Belfield, C. (2003). Education privatization: causes, consequences, and planning implications. París: International Institute for Educational Planning of unEsCO.

Lubienski, C. A. y Theule, S. (2014). The public school advantage: why public schools outperform private schools. Chicago: The University of Chicago.

Raphael, R. (2014). El Mirreynato. La otra desigualdad. México: Planeta.

Reforma. (febrero de 2013). Las mejores preparatorias privadas 2013. Reforma, secc. Universitarios.

Secretaría de Educación Pública. (2014). Sistema Nacional de Información Estadística Educativa. Recuperado de http://www.planeacion.sep.gob.mx/

Tiramonti, G. y Ziegler, S. (2008). La educación de las élites. Aspiraciones, estrategias y oportunidades, Buenos Aires: Paidós.

Torres, V. (2004). La educación privada en México, 1903-1976. México: El Colegio de México/Universidad Iberoamericana.

Ziegler, S. y Gessaghi, V. (2012). Formación de las élites: investigaciones y debates en Argentina, Brasil y Francia. Buenos Aires: Manantial-Flacso.

Zweigenhaft, R. (1992). The application of social and cultural capital: a study of the 25th reunion entries of prep school and public school graduates of Yale College. Higher Education, 23(3), 311-20. Recuperado de https://www.jstor.org/stable/3447379

Zweigenhaft, R. (1993). Prep school and public school graduates of Harvard. The Journal of Higher Education 64(2), 211-25. doi:10.1080/00221546.1993.11778423 